This is a postprint version of the following published document:

Martín, H., Peris-Lopez, P., Tapiador, J.E., San Millan, E. (2016). A New TRNG Based on Coherent Sampling With Self-Timed Rings. IEEE Transactions on Industrial Informatics, vol. 12, no. 1, pp. 91-100.

Available in http://10.1109/TII.2015.2502183

(c) XXXX. IEEE. Personal use of this material is permitted. Permission from IEEE must be obtained for all other uses, in any current or future media, including reprinting/republishing this material for advertising or promotional purposes, creating new collective works, for resale or redistribution to servers or lists, or reuse of any copyrighted component of this work in other works. 


\title{
A New TRNG Based on Coherent Sampling With Self-Timed Rings
}

\author{
Honorio Martin, Pedro Peris-Lopez, Juan E. Tapiador, and Enrique San Millan
}

\begin{abstract}
Random numbers play a key role in applica-tions such as industrial simulations, laboratory experimen-tation, computer games, and engineering problem solving. The design of new true random generators (TRNGs) has attracted the attention of the research community for many years. Designs with little hardware requirements and high throughput are demanded by new and powerful applica-tions. In this paper, we introduce the design of a novel TRNG based on the coherent sampling (CS) phenomenon. Contrary to most designs based on this phenomenon, ours uses self-timed rings (STRs) instead of the com-monly employed ring oscillators (ROs). Our design has two key advantages over existing proposals based on CS. It does not depend on the FPGA vendor used and does not need manual placement and routing in the manufacturing process, resulting in a highly portable generator. Our exper-iments show that the TRNG offers a very high throughput with a moderate cost in hardware. The results obtained with ENT, DIEHARD, and National Institute of Standards and Technology (NIST) statistical test suites evidence that the output bitstream behaves as a truly random variable.
\end{abstract}

Index Terms-Coherent sampling (CS), FPGAs, self-timed ring (STR), true random generator (TRNG).

\section{INTRODUCTION}

$\mathbf{S}$ OURCES of random numbers are always in demand. They play a key role in computer games, problem solving techniques in engineering, industrial simulations, security primitives and protocols, and a variety of other applications. In many cases, the quality of the randommess must be as high as possible, e.g., when used in security applications to generate keys, nonces, session identifiers etc., while in others it is also required a very high throughput in the generation process. In this regard, hardware-based pseudo and true random number generators [pseudo-random number generator (PRNG) and true random generator (TRNG), respectively] are very appealing

H. Martin and E. S. Millan are with Department of Electronics Technology, Universidad Carlos III de Madrid, 28911 Madrid, Spain (e-mail: hmartin@ing.uc3m.es; quique@ing.uc3m.es).

P. Peris-Lopeze and J. E. Tapiador are with the Computer Security Laboratory, Department of Computer Science, Universidad Carlos III de Madrid, 28911 Madrid, Spain (e-mail: pperis@inf.uc3m.es: jestevez@inf.uc3m.es). because of their superior perfomance when compared with software implementations [1]-[3].

Motivated by this, many researchers have pointed out the convenience of using fiel programmable gate arrays (FPGAs) as TRNG platforms due to their low cost and versatility [4][6]. However, FPGAs offer a resource-constrained enviromment (fi ed logic blocks) that does not include analog blocks, which are frequently employed to generate very entropic outputs. Thus, the typical phenomena used in the generation of randomness in FPGAs are metastability and jitter [7], [8]. Since FPGAs are initially designed and implemented to reduce their random behavior, it is considerably more challenging to implement a TRNG in an FPGA than in other digital devices.

From the implementation point of view, a TRNG should not be technology dependant. For instance, in [9] it is presented a TRNG that exploits the technique of coherent sampling (CS) and uses an analog phase-locked loop (PLL) to obtain a fin control over the clock signal. In particular, the proposed TRNG is implemented in an Altera FPGA, which includes a PLL. Unfortunately, this design is not portable to the FPGAs of other important manufacturers of the sector, e.g., such as the ones produced by Xilinx, whose FPGAs mainly use delaylocked loops (DLLs) instead of PLLs. Apart from being technological independent, proposed designs should not be device dependant. For example, Kohlbrenner and Gaj present in [10] a TRNG that uses ring oscillators (ROs) and takes advantage of the CS technique. The design consists of two independent and identically configure ROs with similar but not identical frequency. A sampling circuit uses one clock signal to sample the other clock signal. Although the design works well theoretically, Kohlbrenner and Gaj show how not only there is a great variation between the RO frequencies in the same FPGA (7\%) but also between different FPGAs. In other words, the design depends so much on the used device that it has to be manually tuned (placement and routing) for each FPGA implementation.

To the best of our knowledge, one of the firs simple and portable designs of a TRNG suitable for different FPGAs was presented by Sunar et al. in [11]. Nevertheless, this design was rapidly discarded since it suffers fom implementation problems, mostly related to the number of signals handled by the XoR-wee. Moreover, the quality of the raw signal is rather poor and needs postprocessing. In [12], Wold and Tan present an enhanced design based on Sunar et al.'s, which attempt to solve its implementation problems while avoiding the need of a postprocessing stage. This enhanced version proposes a reduction in the number of rings that, as reported in [13]. cause the loss of entropy. However, such a lack of entropy is masked by 
the pseudorandomness caused by XOR-ing clock signals having different frequencies. In addition, these two designs were successfully attacked in [14] and [15] exploiting the use of ROs and their vulnerabilities to frequency injection, in which the ROs are locked to an injected frequency and the jitter phenomena as a source of randomness is neutralized.

More recently, inspired by Sunar $e$ t al.'s design, Cherkaoui et al. [16] have presented a new design in which the ROs are replaced by a self-timed ring (STR). An STR is a multiphase generator that can maintain constant phase difference between the different outputs. Therefore, this construction is resistant to the common vulnerabilities used to attack TRNGs based on ROs. Cherkaoui et al.'s TRNG seems to be a secure design (no attack has been published yet), but the design consumes a substantial amount of resources in terms of power and circuit area. This renders it unsuitable for constrained devices. More specifically, the STR generates 63 signals that are sampled and finally passed through an XOR tree, generating a high activity and, correspondingly, having a high power consumption. Moreover, the hardware requirements are superior to the ones that can be afforded in most constrained devices.

In this paper, we present a new TRNG based on the CS technique. On the one hand, the design takes advantage of some key STR features, which help us to solve the implementation problems (mainly the device dependence) suffered by Kohlbrenner and Gaj's design [10], while simultaneously avoiding the vulnerabilities linked to the use of ROs. On the other hand, the proposed design is very efficient in hardware, which makes it suitable for devices with limited capabilities, and offers a relatively high throughput.

This paper is organized as follows. In Section II, we describe the CS technique. We explain the phenomenon and the randomness extraction technique. An overview of STRs and their operation principles is presented in Section III. In Section IV, our proposal is presented together with some implementation considerations needed. The experimental results, both about the randomness quality and hardware requirements, are presented in Section V, together with a comparison between our proposal and the most relevant designs. Finally, Section VI concludes the paper and summarizes our main contributions.

\section{COHERENT SAMPLING}

In this section, we first introduce the principles of CS. After that, we present the main TRNG proposals that exploit this technique.

\section{A. Background}

CS is a well-known technique to sample periodic signals at finer time intervals. CS refers to an integer number of cycles that fits into a predefined sampling window. Mathematically, this can be expressed as

$$
\underset{f_{\text {sample }}}{f_{\text {in }}}=N_{\text {samples }}
$$

where $f_{\text {in }}$ is the sampled signal $\left(S_{1}\right)$ frequency, $f_{\text {sample }}$ is the sampling signal $\left(S_{2}\right)$ frequency, $N_{\text {cyc }}$ is the number of cycles of the sampled signal, and $N_{\text {samples }}$ is the number of samples.

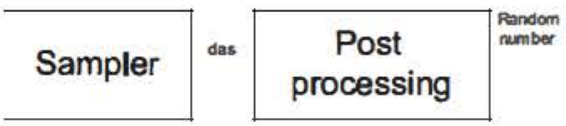

Fig. 1. General architecture of a TRNG based on CS.

If $N_{\text {cyc }}$ and $N_{\text {samples }}$ are high and coprimes, the repetition period of samples will be maximum, i.e., we will have the highest resolution of the sampled signal. This is an interesting feature because if the number of periods (frequencies) is constant for ideal sources of $S_{1}$ and $S_{2}$, in physical systems where these clock signals contain jitter, this number will be random because of the Gaussian random component contained in the jitter.

The general architecture of a TRNG using CS is depicted in Fig. 1. The signal $S_{1}$ would be sampled by the signal $S_{2}$, generating a digitized analog signal known as "das." If the quality of the raw output is not high enough, a postprocessing stage is added to guarantee a uniform output. A mathematical model of physical RNGs based on CS can be found in [17].

\section{B. TRNGs Based on CS}

The first time, to the best of our knowledge, that CS was used in an FPGA to generate random numbers was in [9]. In that work, Fischer and Drutarovsky used a PLL embedded in an Altera FPGA to guarantee the relation between $N_{\text {cyc }}$ and $N_{\text {samples. }}$. As explained in Section I, the main drawback of this proposal is that the TRNG is not portable to other FPGA vendors. Besides, PLLs are not supported in all FPGAs.

In [10], Kohlbrenner and Gaj replaced PLLs by ROs with the aim of obtaining a portable design for FPGAs from different vendors. The RO frequencies are selected to be close but not identical. The RO outputs are connected to a sampler circuit that generates a stream of 0 's and I's. The length of this stream is counted module 2 to generate a random bit. The weakest point of this design is that it requires a very complicated manual placement and routing process to finely set the ring frequencies. According to [10], this is a consequence of the high variation (up to $7 \%$ ) between the RO frequencies in the same FPGA. To overcome such a sensitivity to placement, the authors suggest a design with four ROs that are sampled by a fifth one.

In [18], Cret et al. take up the basic idea of using only two ROs. In this design, the authors introduce a multiplexer to alternate the sampling signal. They claim that the placement sensitivity is overcome using a parametrizable postprocessing. The main weakness of this TRNG is that the quality of the raw output, without the postprocessing stage, is really poor. In addition, Cret et al. present the cycle lengths of the signal generated in the sampler and its distribution is not an evidence of the claimed randomness - which is actually far away from a uniform distribution.

Finally, in [19], the authors present three designs based on different clock generators for different FPGA models. More precisely, the generators are RO-RO, RO-PLL (for Altera FPGAs), and RO-DFS (for Xilinx FPGAs). Apart from the 


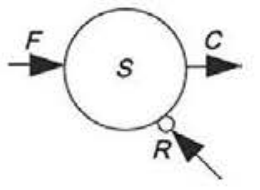

\begin{tabular}{cc|c|}
\hline $\mathrm{F}$ & $\mathrm{R}$ & $\mathrm{C}$ \\
\hline 0 & 0 & $C^{-1}$ \\
0 & 1 & 0 \\
1 & 0 & 1 \\
1 & 1 & $C^{-1}$
\end{tabular}

Fig. 2. Structure and truth table of an STR stage.

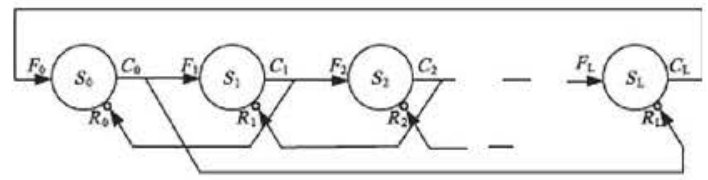

Fig. 3. STR structure.

technology dependency, the pair RO-RO cannot be fully automated since its design needs manual placement and routing. Finally, it is worth mentioning that the authors introduce the interesting idea of generating one random bit per half period by using mutual sampling.

\section{SELF-TIMED RINGS}

STRs are a well-known structure to generate clock signals in digital devices. An STR implements an asynchronous handshake protocol that assures an even distribution of events through the different stages. For this, a multiphase oscillator based on a STR is tuneable by adjusting the frequency and the phase resolution between signals.

\section{A. Architecture}

The basic element in an STR is a stage. Each stage consists of a Muller gate and an inverter, and implements the truth table shown in Fig. 2. If the forward input $F$ is different from the reverse input $R$, the output $C$ takes the same value as $F$; otherwise, the previous output is maintained.

The STR architecture implements a micropipeline (ripple FIFO) introduced by Sutherland in [20] (see Fig. 3). The handshake protocol used guarantees the phase distribution between the micropipeline stages.

\section{B. Behavior and Configuration}

In order to understand the STR operation we need to define the following parameters.

1) $L$ : The number of stages that compose the STR. Each stage can be initialized either to 0 or 1 .

2) Tokens and Bubbles: A stage contains a token if its output $C_{i}$ is not equal to the output $C_{i+1}$. Conversely, a stage contains a bubble if its output $C_{i}$ is equal to the output $C_{i+1}$. The number of tokens (NT) and bubbles (NB) can be chosen during the initialization phase.

3) $N$ : The number of events distributed throughout the ring, which equals the number of propagating tokens in the ring.

$$
\begin{aligned}
& \text { TTTTBBBB }(01010000) \rightarrow \text { TTTBTBBB }(01011000) \rightarrow \\
& \text { TTBTBTBB }(01011000) \rightarrow \text { TBTBТBTB }(01100110) \rightarrow \\
& \text { BTBTBTBT }(11001100) \rightarrow \text { TBTBTBTB }(10011001) \rightarrow \\
& \text { ВТВТВТВТ }(00110011) \rightarrow \text { ТВТВТВТВ }(01100110) \cdots
\end{aligned}
$$

Fig. 4. Example of tokens and bubbles propagation in an STR.

A token will propagate to the next stage $\left(s_{i+1}\right)$ if this stage contains a bubble. The bubble will occupy the backward stage $s_{i}$. The STR will have an oscillatory behavior if there are at least three stages, one bubble, and an even number of tokens. For example, in an eight-stage STR with an initial distribution of four tokens and four bubbles, the events will propagate as shown in Fig. 4.

It is important to note that the propagation delay of the ring depends on two analog phenomena: 1) charlie effects; and 2) drafting effects. The Charlie effect is related to the separation time between events at the inputs: a shorter separation time of events results in a longer stage propagation delay. The drafting effect connects the gate propagation delay with the elapsed time for the output events. According to [21], the drafting effect is negligible in FPGAs. We refer the reader to [22], where a complete model that explain both phenomena and how they affect the ring propagation delay can be found.

As for the configuration possibilities, the frequency can be fine tuned in the initialization phase by changing the ratio between tokens and bubbles, i.e., NT/NB. The maximum frequency is reached when

$$
\frac{\mathrm{NT}}{\mathrm{NB}} \simeq \frac{D_{\mathrm{ff}}}{D_{\mathrm{rr}}}
$$

where $D_{\mathrm{ff}}$ and $D_{\mathrm{rr}}$ are the static forward and static reverse propagation delays, respectively.

The phase shift between two stages separated by $n$ stages can be calculated as

$$
\varphi_{n}=n \times \frac{N}{L} \times 90^{\circ} .
$$

Note that if $L$ is a multiple of $N$, some outputs will have the same phase, as it happens in the example shown in Fig. 4. In particular, there will be four different phases throughout the ring, with each phase appearing in two different stages (stage $_{i}$ and stage $_{i+4}$ ). Thus, for applications of this oscillator in which the goal is typically to generate the maximum number of different phases, $L \bmod N$ should not be equal to 0 .

\section{OUR DESIGN}

The design presented in this paper is inspired by the TRNG proposed by Kohlbrenner and Gaj in [10]. We use the same architecture but replace the ROs by two STRs. Cherkaoui et al. carried out in [21] an exhaustive comparison between ROs and STRs. According to this work, the main differences are as follows. 


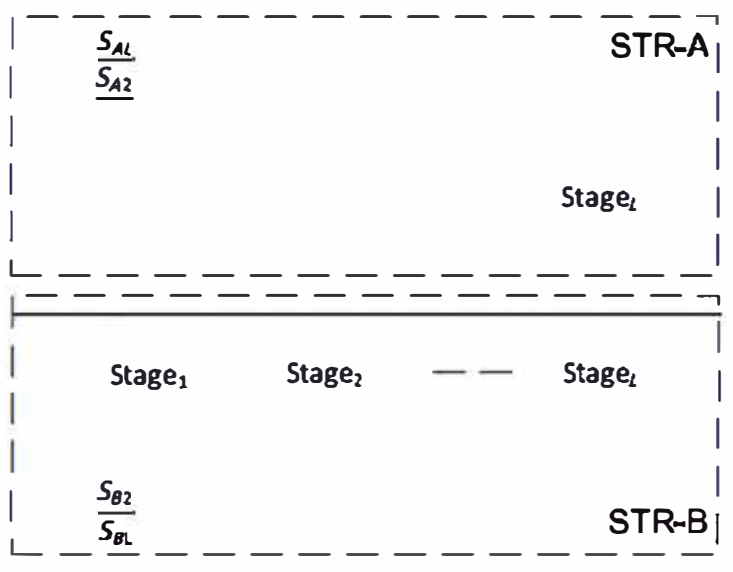

Fig. 5. STR structure of our TRNG.

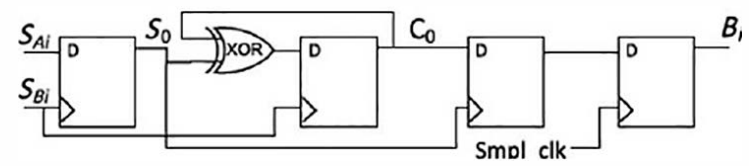

Fig. 6. Sampler structure of our TRNG.

1) STR robustness to voltage variations can be enhanced by adding more stages. ROs do not offer this feature.

2) STRs present a lower extra-device frequency variation when operating at high frequencies.

3) In STRs, the period jitter does not depend on the number of stages but it is mostly dependant on the jitter generated at each stage.

From the security point of view, these features are very interesting. In fact, in [21], the authors conclude that STRs are more robust to attacks than ROs and this property is inherited by our proposal. Furthermore, replacing ROs by STRs provides our design with the possibility of having at least $L$ different signals in each STR. Each one of those $L$ signals can be used as a sampling or sampled signal, since each stage can be considered as an independent source of entropy [16], the number of stages is equal to the number of independent entropy sources. Moreover, STRs are highly configurable. In particular, it is very easy to set the desired frequency for the STR output, which allows a fine-grained control over the resulting speed of the TRNG.

\section{A. Architecture Overview}

Figs. 5 and 6 show the different blocks that make up our TRNG. Fig. 5 depicts the two STRs used in our design. Both STRs are composed of $L$ stages that generate $L$ different outputs with a frequency $f_{S T R}$. The number of tokens and bubbles are selected in the reset phase attending to the frequency and phase necessities.

The jitter contained in the STR outputs is extracted using the sampler circuit shown in Fig. 6. Each sampler circuit is composed of four-dimensional (4-D)-type flip-flops and one XOR gate. The first flip-flop uses the signal $S_{B i}$ to sample the signal $S_{A i}$. The signal $S_{0}$ will be high while the rising edges of $S_{B i}$ occur during the high level of $S_{A i}$. In Fig. 7, we show the behavior of $S_{0}$ taking into account that $S_{B i}$ contains jitter. As

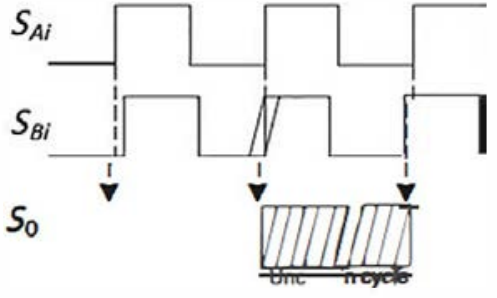

Fig. 7. Sampler behavior.

consequence of such a jitter, the cycle length of $S_{0}$ will not be constant.

In our design, both signals $S_{B i}$ and $S_{A i}$ contain jitter. As a variation of the original sampler design that includes a 1-bit counter latched by $S_{0}$, in our design, we use the simplified version presented in [19]. In this scheme, instead of counting the cycles of $S_{B i}$, we count the number of cyclesthat $S_{0}$ is at a high level. If such a number of cycles is even, the previous output is maintained; othenwise, the output changes. Two D flip-flops and one XOR gate are involved in this process. Finally, the last flipflop samples the signal $C_{0}$ using an external clock. This external clock determines the TRNG throughput. As our design is composed of two STRs with $L$ stages each, $L$ sampler circuits are necessary (see Fig. 1).

Finally, our design includes a postprocessing unit that might be needed depending on the quality, in terms of randomness, of the raw data. The selected postprocessing is a parity filter, which has been widely used as postprocessing in previous proposals such as [16] and [18]. More precisely, an $n$th parity filter takes $n$ consecutive bits and XOR all of them together to produce one bit. This postprocessing offers a simple bias reduction with the penalty of a throughput reduction-the filter reduces the bit generation by a factor of $n$.

\section{V.EXPERIMENTALRESULTS}

In order to evaluate the portability of our proposal, we have implemented our design on FPGAs from three different manufacturers: 1) a Spartan-3E XC3S500E FPGA from Xilinx; 2) an Igloo M IAGL 1000 from Microsemi; and 3) a Cyclone II EP2C5F256C8 from Altera. As expected, the obtained results are similar in all of them. In addition, to show the independence of our design from the manufacturing technology, we have also implemented one final chosen design on another two different FPGAs that use different process technologies: 1) a Virtex 5 XC5VLX110T (65 nm); and 2) a Virtex 6 XC6VLX240T $(40 \mathrm{~nm})$ from Xilinx. In the following, we discuss our results in detail.

Two eight-stage STRs have been implemented and configured in the reset phase to obtain an STR output frequency of $300 \mathrm{MHz}$. Several frequencies have been used in the external clock that samples the signal $C_{0}$. Eight bits are generated with each rising edge of the sampling clock.

In order to obtain almost the same propagation delay in the different stages, a hard macro (or its equivalent for other FPGA vendors) has been designed. This hard macro implements a Muller gate and an inverter using a single look-up table (LUT). 

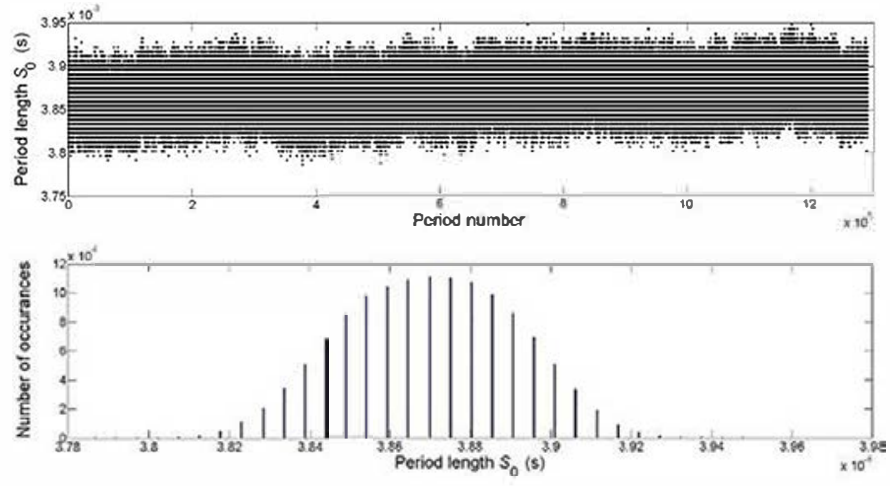

Fig. 8. Time evolution and histogram of $S_{0}$.

We have chosen two STRs with eight stages since this configuratio is easily tunable and offers a good tradeoff between area and throughput. In addition, this configuratio allows the generation in parallel of 8 bits (1 Byte), which is a typical bit length used in many applications. The throughput goal has been set to $1 \mathrm{Mb} / \mathrm{s}$ to be comparable to other TRNGs proposals based on CS. This throughput threshold will set the lowest sampling frequency that can be used in our design.

\section{A. Testing Randomness}

In this section, we discuss the quality of the TRNG output in temms of randomness. Following the standard practice in this field we firs show that the STR outputs have Gaussian jitter and then report the results obtained with three widely used suites of statistical tests for cryptographic applications. We have also carried out a restart experiment to provide evidence that the output is different after repeatedly restarting the system under the same conditions.

Due to space limitations, all results reported in this section correspond to traces obtained with the Spartan-3E XC3S500E FPGA. The conclusions for the other four FPGAs are identical to those shown here.

1) Evidence of the Gaussian Jitter: In order to show evidence of the presence of Gaussian jitter in the STR outputs, we have counted the number of cycles of the signal $S_{0}$, as done in [19] and [10]. Fig. 8 depicts the time evolution of the $S_{0}$ length (top) and a histogram of the cycles (bottom). The his-togram population corresponds with $1.3 \times 10^{6}$ measurements. The average period of $S_{0}$ is $38.69 \mathrm{~ns}$ with an standard devia-tion of $0.215 \mathrm{~ns}$. As the frequency of the STR has been set to 300 $\mathrm{MHz}$, which means that the average cycle length is 11.61 cycles. In conclusion, the histrogram distribution clearly shows evidence of the underlying randomness in the sampling process, and by extension, in each stage of the STR.

2) Restart Experiment: Following the same procedure used in [12] and [23] to distinguish the amount of true randomness contained in a pseudorandom oscillating signal, we have carried out a restart experiment. In Fig. 9, nine oscillograms of repeated restarts from identical starting conditions are presented. The horizontal axis represents the time and shows the firs 20 bits generated after each restart (the period of time shown for each restart is $20 \mu \mathrm{s}$ using a sampling clock of
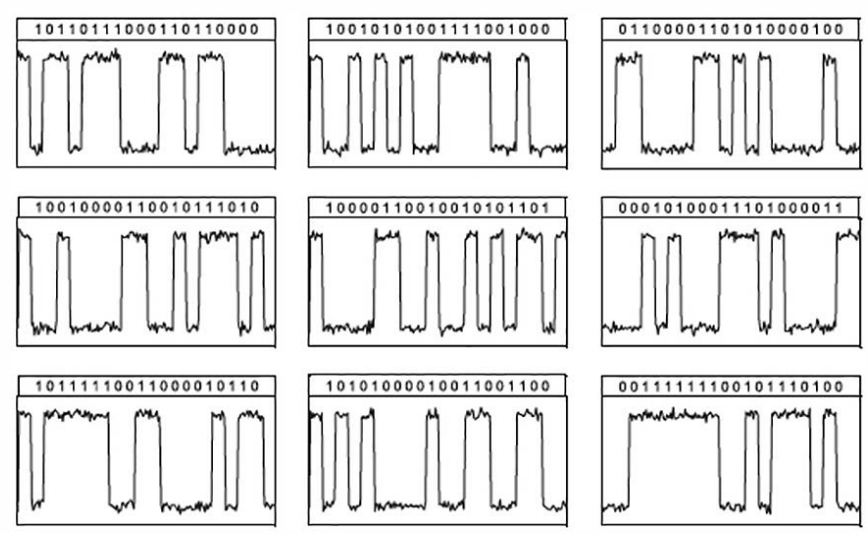

Fig. 9. Nine output sequences captured after restarting the TRNG. Note that all sequences are different.

$1 \mathrm{MHz}$ ). The vertical axis is the voltage of the output signal. Only nine curves out of the 1000 generated are shown. It is clear that the TRNG generates different traces after the same restarting point.

3) Statistical Evaluation of the Output: The testing of our proposal has been carried out using the NIST statistical test suite [24], as commonly done to validate previous proposals (e.g.. [9], [10], [18]). To ransfer the bits generated by the TRNG in the FPGA to the host computer where the NIST tests are executed, a FIFO memory and an RS232 communication protocol have been used. In addition, the postprocessing has been conducted in the host computer in order to reduce the acquisition time of the traces.

We have evaluated the TRNG output for the following set of sampling frequencies: $50,25,10,5,1$, and $0.5 \mathrm{MHz}$ ). A higher sampling frequency will imply a higher throughput, but also a lower quality of the random bits due to the fact that the jitter accumulation time is shorter. According to the study presented in [25], a longer accumulation time is desirable so that the contribution of the thermal noise (responsible of the nondeteministic jitter) is perceptible. On the other hand, the use of a longer accumulation time causes that the flic er noise (responsible of the deterministic jitter) dominates the jitter. This paradox forces designers to fin a tradeoff to set the sampling frequency.

For the postprocessing, we have tested the minimum parity filte order (bit-wise XOR tree) necessary to pass the NIST tests for the different sampling frequencies studied. A third-order fil ter is needed for $50 \mathrm{MHz}$, while a second-order filte suffice for the rest. As expected, the postprocessing necessities are higher when higher sampling frequencies are used. Although many sampling frequencies need the same order parity fil ter, it is important to notice that the proportion of failed tests before the postprocessing rises when the sampling frequency is increased, as explained below. This is a crucial point if for some reason the TRNG will be used without the postprocessing block.

We have evaluated the quality of the raw data before the postprocessing for the six sampling frequencies studied. Fig. 10 shows boxplots of the $p$-value distribution for each șampling 


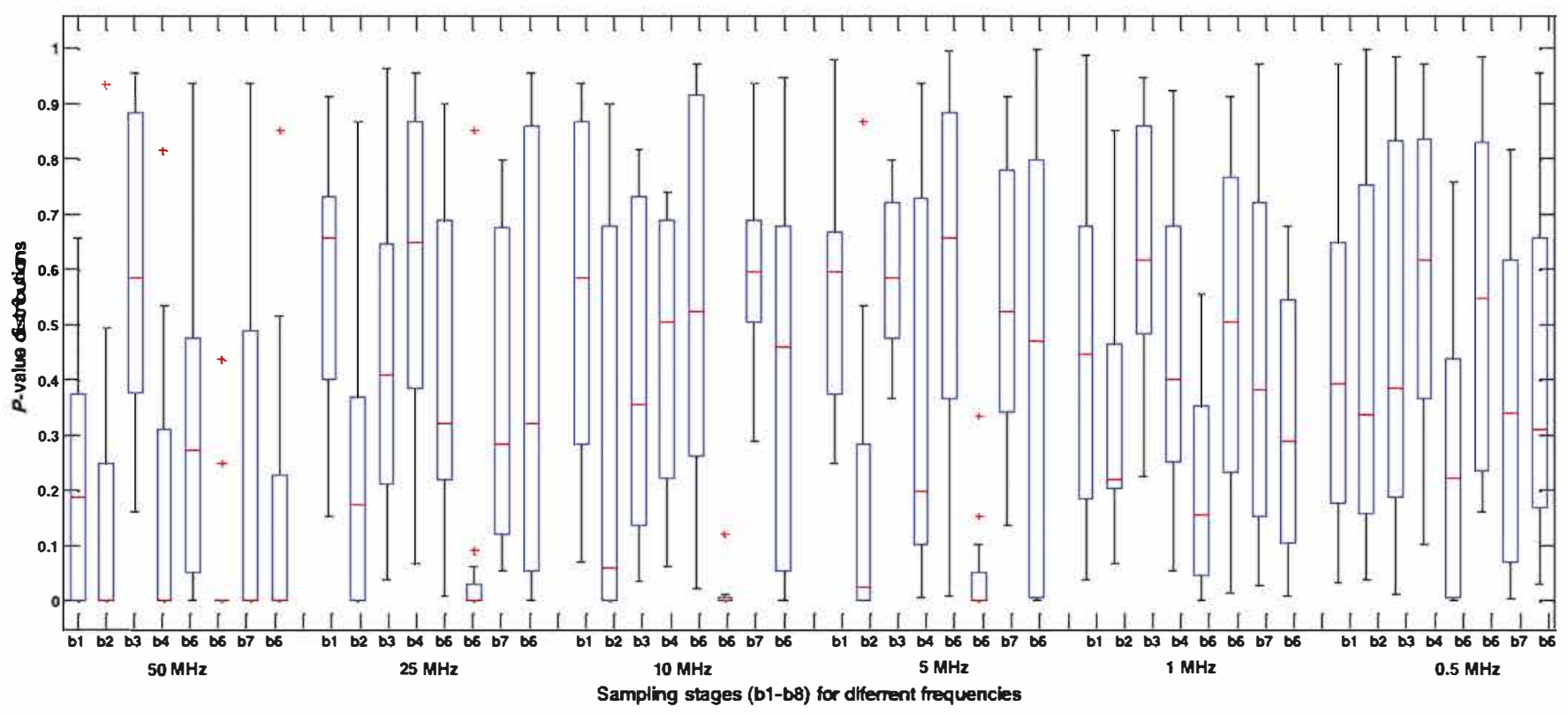

Fig. 10. Boxplots of $p$-value distributions for each sampling stage ( $b 1$ to $b 8$ ) and different frequencies.

TABLE I

Experimental Results: Pass Rate (PR) Proportion and Average p-Value (PV) for Generated traces

\begin{tabular}{|c|c|c|c|c|c|c|c|c|c|c|c|c|}
\cline { 2 - 13 } \multicolumn{1}{c|}{} & \multicolumn{2}{c|}{ 0.5 MH7. } & \multicolumn{2}{c|}{ I MH7. } & \multicolumn{2}{c|}{$5 \mathrm{MHz}$} & \multicolumn{2}{c|}{ 10 MH7. } & \multicolumn{2}{c|}{$25 \mathrm{MH7}$} & \multicolumn{2}{c|}{$50 \mathrm{MHz}$} \\
\cline { 2 - 14 } \multicolumn{1}{c|}{} & PR & PV & PR & PV & PR & PV & PR & PV & PR & PV & PR & PV \\
\hline b1 & 97.91 & 0.41 & 98.58 & 0.44 & 98.41 & 0.57 & 99.08 & 0.55 & 99.5 & 0.58 & 93.83 & 0.22 \\
b2 & 98.41 & 0.43 & 99.33 & 0.34 & 82.58 & 0.18 & 82.5 & 0.30 & 84.66 & 0.22 & 39.66 & 0.16 \\
b3 & 98.83 & 0.46 & 99.08 & 0.62 & 98.58 & 0.59 & 98.91 & 0.41 & 98 & 0.44 & 99.33 & 0.60 \\
b4 & 99.66 & 0.59 & 99.41 & 0.45 & 99.33 & 0.36 & 99.41 & 0.45 & 99 & 0.59 & 83.41 & 0.17 \\
b5 & 92.66 & 0.25 & 87.16 & 0.20 & 98.5 & 0.59 & 98.25 & 0.55 & 99.16 & 0.39 & 97.91 & 0.30 \\
b6 & 98.41 & 0.54 & 98.75 & 0.47 & 31.66 & 0.04 & 31.58 & 0.01 & 31.25 & 0.08 & 22.25 & 0.05 \\
b7 & 98.75 & 0.36 & 99.16 & 0.45 & 98.83 & 0.53 & 98.91 & 0.61 & 98.58 & 0.36 & 66.58 & 0.24 \\
b8 & 99.16 & 0.39 & 98.91 & 0.31 & 98 & 0.44 & 97.5 & 0.40 & 96.83 & 0.43 & 48.5 & 0.15 \\
\hline Tolal & 97.97 & 0.43 & 97.55 & 0.41 & 88.23 & 0.41 & 88.27 & 0.41 & 88.37 & 0.39 & 68.93 & 0.24 \\
\hline
\end{tabular}

stage (b1-b8) and different frequencies. According to the documentation provided by NIST, a random stream must present uniformity in the distribution of its $p$-values. It can be seen in Fig. 10 that higher sampling frequencies presents less uniformity for its $p$-values distribution than lower sampling frequencies, which are more uniform.

Further evidence of this phenomenon is presented in Table I, which shows the proportion of traces that pass the statistical tests (PR) and the average $p$-value (PV) for the different sampling stages and frequencies. Note that traces corresponding to $b 5$ and $b 6$ perform quite badly, specially $b 6$. For sampling frequencies of 0.5 and $1 \mathrm{MHz}$, only a single trace (b5) fails the NIST tests before the postprocessing. It is noteworthy, however, that $b 5$ fails the tests by a narrow margin. Three traces of $b 5$ fail the tests for the sampling frequencies of 5,10 , and $25 \mathrm{MHz}$, and seven aces fail for $50 \mathrm{MHz}$. As for $b 6$ traces, they fail badly for the sampling frequencies between 5 and $50 \mathrm{MHz}$. This consistent behavior in $b 6$ is mainly due to the fact that the synthesizer has placed the sampling stage that generates the $b 6$ stream in a way that causes a huge delay between the sampling $\left(S_{A 6}\right)$ and the sampled $\left(S_{B 6}\right)$ signals. This problem could be solved using a manual placement and routing process. In fact, we have tested this using manual placement and routing and setting the sampling frequency to $50 \mathrm{MHz}$ results in a design such that the raw stream of bits without postprocessing passes the NIST tests. Nevertheless, one major design goal of our proposal is to avoid such a manual procedures.

We have evaluated the quality of our proposed TRNG after postprocessing. A sampling frequency of $1 \mathrm{MHz}$ has been selected for this experimentation since this frequency offers a tradeoff between throughput and randomness quality before the postprocessing stage. We have opted for having a good quality signal without postprocessing to make stronger our TRNG proposal against some attacks. ENT [26], DIEHARD [27], NIST [24], and AIS31 [28] suites have been used for analyzing the randommess quality.

In Table II, we summarize the results obtained with ENT, which resemble those obtained with a genuine random variable, such as the chi-square test is passed, entropy is extremely high, the serial correlation is very low, etc. DIEHARD is a much more demanding battery of tests for checking randomness. As in the case of the NIST suite, DIEHARD is particularly 
TABLE II

ENT RESULtS FOR A SAMPLING FREQUENCY SET TO $1 \mathrm{MHZ}$

\begin{tabular}{|l|c|}
\hline Entropy & 7.999987 \\
\hline Compression & $0 \%$ \\
\hline Chi square distribution & $261.92(36.96 \%)$ \\
\hline Arithmetic mean & 127.5110 \\
\hline Monte Carlo value for $\mathrm{Pi}$ & 3.141718075 \\
\hline Serial correlation coefficient & 0.000368 \\
\hline
\end{tabular}

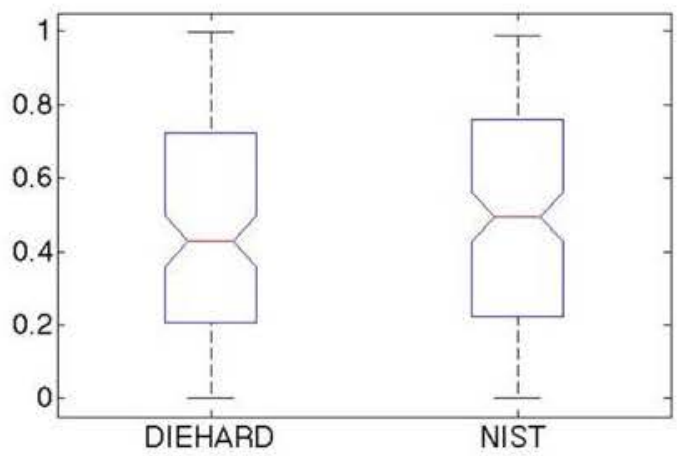

Fig. 11. Distribution of $p$-values for the DIEHARD and NIST test suites.

TABLE III

AIS31 RESULTS FOR THREE FPGAS

\begin{tabular}{|c|c|c|c|c|}
\hline \multirow{2}{*}{ Device } & \multirow{2}{*}{ Frequency $(\mathrm{MHz})$} & \multicolumn{2}{|c|}{ Rawdata } & Postprocessing \\
\cline { 2 - 5 } & & T1-T4 & T5-T8 & $n_{\text {pmin }}$ \\
\hline \multirow{2}{*}{ Spartan-3E } & 1 & $4 / 4$ & $3 / 4$ & 2 \\
\cline { 2 - 5 } & 50 & $3 / 4$ & $3 / 4$ & 3 \\
\hline \multirow{2}{*}{ Igloo } & 1 & $4 / 4$ & $4 / 4$ & - \\
\cline { 2 - 5 } & 50 & $4 / 4$ & $3 / 4$ & 2 \\
\hline \multirow{2}{*}{ Cyclone II } & 1 & $4 / 4$ & $3 / 4$ & 2 \\
\cline { 2 - 5 } & 50 & $3 / 4$ & $3 / 4$ & 3 \\
\hline
\end{tabular}

designed for cryptographic applications and includes a number of statistical tests (e.g., frequency, rank, fft, monkey, runs, and so on). A fina $p$-value is obtained for each test. If we take a significanc level of $0.05, p<0.025$ or $p>0.975$ means that the TRNG fails the test. To show evidence that our proposed TRNG behaves as a random variable, in Fig. 11, we depict the distribution of $p$-values for all tests included in both suites. In particular, all the $p$-values in the NIST and DIEHARD suites are within the interval $[0.2,0.8]$, so the TRNG passes all tests in both suites.

Finally, we have evaluated the data acquired from the three FPGAs using the AIS31 statistical test suite. Using two sampling frequencies of 50 and $1 \mathrm{MHz}$, we have gathered a 1-MB sequence of raw data. The results for the AIS31 statistical suite are depicted in Table III. Note that tests $T 1-T 4$ correspond to four FIPS 140-1 tests (poker, monobit, runs, and long runs). $T 5$ is an autocorrelation test, $T 6$ is a uniform distribution test, $T 7$ is a comparative test for multinomial distributions, and $T 8$ is an entropy test. According to the AIS31 recommendations, raw data from the TRNG output or at least data at the output of the arithmetic postprocessing, should pass $T 5$ through $T 8$. The column $n_{\text {pmin }}$ represents the minimum filte order to comply with this requirement. For the AIS 31 results - as shown in
TABLE IV

HARDWARE RESOURCES

\begin{tabular}{|l|l|}
\hline FPGA & Hardware resources \\
\hline Cyclone II EP2C5F256C8 & 43 LUTs and 48 Registers \\
\hline Igloo M1AGL1000 & 93 Core Cells (48 DFFN) \\
\hline Spartan-3E XC3S500E & 32 LUTs and 48 Registers \\
\hline \hline Virtex 5 XC5VLX110T & 32 LUTs and 48 Registers \\
\hline Virtex 6 XC6VLX240T & 32 LUTs and 48 Registers \\
\hline
\end{tabular}

Table III and, equivalently, for NIST $p$-values in Fig. 10-we have obtained better results for lower frequencies.

From all of the above, we can conclude that our TRNG outputs a bit stream that looks like a true random variable.

\section{B. Hardware Resources}

The results presented in this section correspond to our chosen design with a sampling frequency of $1 \mathrm{MHz}$. The architecture consists of two eight-stage STRs, eight sampling stages, and a second-order parity filte as postprocessing block.

Since each STR stage uses a single LUT, the STRs occupies $2 \times L$ LUTs. As shown in Fig. 6, the sampler structure uses four registers and an XOR gate (one LUT). Therefore, the number of LUTs used by the sampler structure is $L$ and the number of reg-isters is $4 L$. Finally, the postprocessing requirements depend on the parity filte of order $n$. The LUTs used by the postpro-cessing is also conditioned by the inputs of each LUT. Since a four-input Xor gate, as in the case of twoinput XOR gates, can be implemented using one LUT, the number of LUTs, and reg-isters will be $L$ and $n L$, respectively - the filte order is 2 for $1 \mathrm{MHz}$ sampling frequency, as explained in Section V-A3.

In summary, observing the results above we can conclude that each raw random bit (before postprocessing) has a cost of three LUTs and four registers. Therefore, for a given sampling frequency ( $f_{\text {sampling }}$ ), a designer could improve the throughput by adding more stages to the STRs. This will result in $f_{\text {sampling }}$ bps per additional stage. On the other hand, this improvement translates into a circuit area penalty of three LUTs and four registers per additional stage.

Table IV summarizes the amount of resources needed to implement our TRNG on fiv different FPGAs. The differences in terms of the combinational logic elements for the set of FPGAs analyzed are related to the optimizations carried out by the different synthesis tools. Note that the same amount of hardware resources are obtained for the three Xilinx FPGAs (i.e., Spartan and Virtex). This is due to the fact that we have tailored the hard macro created for the Spartan-3E to fi into the Virtex 5 and 6. It is important to emphasize the decision of implementing each STR stage in a single LUT to have almost the same delay between consecutive stages to avoid bottleneck effects.

Regarding throughput, our proposal is able to generate random bits in parallel. For the proposed architecture, eight random bits are generated each two clock cycles. This feature can be very interesting for some applications that require the generation of random bits in parallel. 
TABLE V

TRNG COMPARISON

\begin{tabular}{|c|c|c|c|c|c|}
\hline & & Hardware resources & Throughput & Hardware complexity & Portability \\
\hline \multicolumn{2}{|c|}{ Our proposal } & 32 LUTs 48 Registers & $4 \mathrm{Mb} / \mathrm{s}$ & Medium & Yes \\
\hline \multicolumn{2}{|c|}{ Fischer ef al. [9] } & 121 LCs + ESBs and I PLL & $69 \mathrm{~Kb} / \mathrm{s}$ & Medium & No \\
\hline \multicolumn{2}{|c|}{ Kohl et al. $\mid 10]$} & 12 LUTs 24 Registers & $3(0) \mathrm{Kb} / \mathrm{s}$ & High & Yes \\
\hline \multirow[t]{3}{*}{ Valtchanov e' al. [19] } & RO-RO & 15 LUTs 4 Registers & $2 \mathrm{Mb} / \mathrm{s}$ & High & Yes \\
\hline & RO-PLL & 12 LCs + Registers and I PLL & $2 \mathrm{Mb} / \mathrm{s}$ & Medium & No \\
\hline & RO-DFS & 11 LUTs 6 Registers and 2 DFS & $2 \mathrm{Mb} / \mathrm{s}$ & Medium & No \\
\hline \multicolumn{2}{|c|}{ Cherkaoui et al. [16] } & 320) LUTs 320) Registers & $2(0) \mathrm{Mb} / \mathrm{s}$ & Medium & Yes \\
\hline
\end{tabular}

\section{Comparison With TRNGs Based on CS}

Next, we present a comparison between our proposal and other TRNG designs that use CS. We also include the proposal of Cherkaoui et al. [16] since it is based on STRs. For each proposal, we have analyzed the hardware resources needed and the offered throughput. In addition, we have also considered the hardware complexity, including the degree of automation of the design, and its portability (device independence). Regarding hardware complexity, we distinguish three categories.

1) Low complexity is devoted for designs that can be easily implemented.

2) Medium complexity implies designs that need to use hard macros or specifi components like PLL or DFS.

3) High complexity considers designs that require a manual place and route process.

Finally, the portability aspect represents whether the design needs special resources or efforts to be implemented in different FPGA vendors or devices.

We emphasize here that the hardware results presented in Table $\mathrm{V}$ constitute an estimation for the designs in which the authors do not provide specifi results. For Cherkaoui et al.'s proposal, we selected the architecture that implements 255 stages.

Table $\mathrm{V}$ shows the comparison between our design and other TRNG proposals. It can be noted that our proposal offers a very good radeoff between the set of parameters evaluated. TRNGs that need a complicated place and route process (e.g.., [1 0$]$ and RO-RO [19]) are superior in terms of hardware resources, but these designs have the drawback of requiring a specifi design for each particular device. Among the TRNGs based on CS, our design offers the highest throughput. Note that this could be even better if a higher sampling frequency would have been selected, although this might degrade the quality of the random signal. On the other hand, Cherkaoui et al. 's TRNG presents the highest throughput, but uses around 1 0 times more resources than our proposal. As aforementioned, in terms of throughput our TRNG generates eight random bits in parallel. Finally, it is worth mentioning that our proposal is highly portable and complies with the two requirements set in Section I: i.e., our design is technology and device independent.

\section{Comparison With Other FPGA-Based TRNGs}

In Section V-C, we have carried out an exhaustive comparison between our proposal and other TRNGs based on CS. Now, we present a qualitative comparison with other state-of-the-art
TRNGs implemented on FPGAs that present some interesting metrics.

Among the several proposals reported in this field the TRNG proposed by Varchola and Drutarovsky [29] stands out because of its lightweightness. This design takes advantage of the metastability on a new bi-stable structure-transition effect ring oscillator (TERO). In terms of area (two CLBs), this TRNG is more lightweight than our proposal but present a very poor throughput $(250 \mathrm{~Kb} / \mathrm{s})$. In addition, experiments show that proper placement and routing strategies are essential.

Exploiting some features of embedded RAMs has become a popular principle nowadays because of the high throughput that can be achieved. Among the proposals that take advantage of SRAMs, those that use write collisions to extract entropy are worth mentioning [30], [31]. The key idea here consists of generating a conflic in a particular address by trying to write opposite values at the same time. In comparison with our TRNG, these proposals present better results in terms of area and throughput, but their portability constitutes a handicap because an enrolment process is necessary in order to identify distinctive BRAMs in each FPGA.

Another interesting proposal based on high fanout nets was presented by Cret et al. in [32]. Its performance is remarkable regarding portability and its high throughput $(60 \mathrm{Mb} / \mathrm{s})$. However, in terms of area, our proposal outperforms this design. Very recently, Wieczorek presented a new FPGA-based TRNG [33] that offers metrics similar to those of our design. The portability of this TRNG is currently under study because only a Xilinx implementation has been reported. Another interesting work is the complementary design proposed in [34], which outperforms ours in terms of throughput but whose portability has not been deeply studied since the results are only validated on a Virtex-6 FPGA. Furthermore, the design in [34] includes a place and route procedure to guarantee the TRNG randomness, which implies some extra effort for the designer when implementing the TRNG in different FPGAs.

All in all, our TRNG presents an attractive tradeoff among hardware footprint, throughput, and portability when compared with existing proposals.

\section{CONCLUSION}

There is a wide set of applications, ranging fiom security services to simulations, computer games, and problem solving tools, where pseudorandom number generators play a central role. In many cases, as a consequence of the high throughput 
required and the high-quality randomness demanded, the use of software-based solutions is simply infeasible. Motivated by this, many FPGA-based proposals have appeared recently.

TRNGs in FPGAs mainly exploit metastability and jitter phenomena as sources of randomness. In this paper, we have proposed a TRNG based on CS, which is a phenomenon that seems to provide good results in previous proposals. Most previous works based on CS rely on either a PLL or an RO. The use of these components has two major drawbacks.

1) It makes the design dependent of the FPGA vendor, for instance, not all FPGA vendors support PPLs.

2) It requires manual placement and routing for setting particular frequencies for each device.

To avoid these two drawbacks, we have proposed a novel design where ROs or PLLs are replaced by STRs. We argue that the use of STRs is very convenient, because it provides robustness against frequency and voltage variations while simultaneously offering one independent source of entropy for each ring stage. Thus, the resulting TRNG combines the power of CS and the robustness and portability linked to STRs. Furthermore, our design does not depend on the FPGA vendor, and the placement and routing is performed automatically by the synthesis tool.

Our proposal outperforms all previous TRNGs based on CS and its throughput could be further increased if we relax our requirements about the quality of the random signals before the postprocessing (e.g., for non-cryptographic applications). We have studied in detail the most restrictive design with a sampling frequency set to $1 \mathrm{MHz}$. In terms of randomness, our TRNG passes all batteries of tests for checking the randomness of a random number generator (ENT, DIEHARD, and AIS31), and also others like NIST that are devoted to evaluate generators designed for cryptographic applications.

\section{REFERENCES}

[1] S. Saab, J. Hobeika, and I. Ouaiss, "A novel pseudorandom noise and band jammer generator using a composite sinusoidal function," IEEE Trans. Signal Process., vol. 58, no. 2, pp. 535-543, Feb. 2010.

[2] J.-L. Danger, S. Guilley, and P. Hoogvorst, "High speed true random number generator based on open loop structures in FPGAS," Microelectron. J., vol. 40, no. 11, pp. 1650-1656, 2009.

[3] R. Vaidyanathaswami and A. Thangaraj, "Robustness of physical layer security primitives against attacks on pseudorandom generators," IEEE Trans. Commun., vol. 62, no. 3, pp. 1070-1079, Mar. 2014.

[4] X. Fang, Q. Wang, C. Guyeux, and J. M. Bahi, "Fpga acceleration of a pseudorandom number generator based on chaotic iterations," J. Inf. Sec. Appl., vol. 19, no. 1, pp. 78-87, 2014.

[5] D. B. Thomas and W. Luk, "The lut-sr family of uniform random number generators for FPGA architectures," IEEE Trans. Very Large Scale Integr. (VLSI) Syst., vol. 21, no. 4, pp. 761-770, Apr. 2013.

[6] P. Wieczorek, "Dual-metastability FPGA-based true random number generator," Electron. Lett., vol. 49, no. 12, pp. 744-745, Jun. 2013.

[7] D. Lubicz and N. Bochard, "Towards an oscillator based trng with a certifie entropy rate," IEEE Trans. Comput., vol. 64, no. 4, pp. 1191-1200, Apr. 2015.

[8] V.B. Suresh and W. Burleson, "Entropy extraction in metastability-based trng," in Proc. IEEE Int. Symp. Hardware-Oriented Sec. Trust (HOST), Jun. 2010, pp. 135-140.

[9] M. Fischer and V. Drutarovsky, "True random number generator embedded in reconfigurabl hardware," in Proc. Int. Workshop Cryptogr. Hardware Embedded Syst. (CHES'02), 2002, vol. 2523, pp. 415-430.

[10] P. Kohlbrenner and K. Gaj, "An embedded true random number generator for FPGAS," in Proc. 12th Int. Symp. Field Programm. Gate Arrays (ACM/SIGDA'04), 2004, pp. 71-78.
[11] S. Sunar, W. J. Martin, and D. R. Stinson, "A provably secure true random number generator with built-in tolerance to active attacks, "IEEE Trans. Comput., vol. 58, no. 1, pp. 109-119, Jan. 2007.

[12] K. Wold and C. H. Tan, "Analysis and enhancement of random number generator in FPGA based on oscillator rings," Int. J. Reconfi . Comput., vol. 2009 , pp. 4:1-4:8, 2009.

[13] N. Bochard, F. Bernard, V. Fischer, and B. Valtchanov, "True-randomness and pseudo-randomness in ring oscillator-based true random number generators," Int. J. Reconfi . Comp., vol. 2010, 2010, Article ID 879281 [Online]. Available: $\mathrm{http} / / / \mathrm{dx}$.doi.org/10.1155/2010/879281

[14] P. Bayon et al., "Contactless electromagnetic active attack on ring oscillator based true random number generator," in Proc. 3rd Int. Workshop Construct. Side-Channel Anal. Secure Des. (COSADE), 2012, pp. 151166.

[15] A. T. Markettos and S. W. Moore, "The frequency injection attack on ring-oscillator-based true random number generators," in Proc. 11th Int. Workshop Cryptogr. Hardware Embedded Syst., 2009, pp. 317-331.

[16] A. Cherkaoui, V. Fischer, L. Fesquet, and A. Aubert, "A very high speed true random number generator with entropy assessment," in Proc. Int. Workshop Cryptogr. Hardware Embedded Syst. (CHES'13), 2013, vol. 8086, pp. 179-196.

[17] F. Bernard, V. Fischer, and B. Valtchanov, "Mathematical model of physical RNGS based on coherent sampling," Tatra Mt. Math. Publ., vol. 45, pp. 1-14, 2010.

[18] O. Cret, A. Suciu, and T. Gyorfi "Practical issues in implementing TRNGS in FPGAS based on the ring oscillator sampling method," in Proc. 10th Int. Symp. Symbol. Numer. Algorithms Sci. Comput. (SYNASC'08), 2008, pp. 433-438.

[19] B. Valtchanov, V. Fischer, and A. Aubert, "Enhanced TRNG based on the coherent sampling," in Proc. 3rd Int. Conf. Signals Circuits Syst. (SCS'09), 2009, pp. 1-6.

[20] I. E. Sutherland, "Micropipelines," ACM Commun., vol. 32, no. 6, pp. $720-738,1989$.

[21] A. Cherkaoui, V. Fischer, A. Aubert, and L. Fesquet, "Comparison of selftimed ring and inverter ring oscillators as entropy sources in FPGAS," in Proc. Des. Autom. Test Eur. Conf. Exhib. (DATE'12), 2012, pp. 13251330.

[22] A. Winstanley and M. Greenstreet, "Temporal properties of self-timed rings," in Correct Hardware Design and Verificatio Methods, vol. 2144. New York, NY, USA: Springer, 2001, pp. 140-154.

[23] M. Dichtl and J. D. Golic, "High-speed true random number generation with logic gates only," in Cryptographic Hardware and Embedded Systems (CHES), vol. 4727, P. Paillier and I. Verbauwhede, Eds. New York, NY, USA: Springer, 2007, pp. 45-62

[24] A. Rukhin et al., "A statistical test suite for random and pseudorandom number generators for cryptographic applications," Natl. Inst. Stand. Technol., Gaithersburg, MD, USA, Tech. Rep., 2010 [Online]. Available: http://csrc nist.gov/rng/

[25] P. Haddad, Y. Teglia, F. Bernard, and V. Fischer, "On the assumption of mutual independence of jitter realizations in P-TRNG stochastic models," in Proc. Des. Autom. Test Eur. Conf. Exhib. (DATE'14), 2014, pp. 1-6.

[26] J. Walker. (1998). Randomness Battery [Online]. Available $\mathrm{http} / / / \mathrm{www}$. fourmilab.ch/random/

[27] G. Marsaglia. (1996). The Marsaglia Random Number CDROM Including the Diehard Battery of Tests of Randomness [Online]. Available: http://stat.fsu.edu/pub/diehard

[28] W. Schindler and W. Killmann, "Evaluation criteria for true (physical) random number generators used in cryptographic applications," in Proc. Revised Papers 4th Int. Workshop Cryptogr. Hardware Embedded Syst., 2003, pp. 431-449 [Online]. Available: $\mathrm{http} / / / \mathrm{dl}$.acm.org/citation. $\mathrm{cfm}$ ?id=648255.752732

[29] M. Varchola and M. Drutarovsky, "New high entropy element for fpga based true random number generators," in Cryptographic Hardware and Embedded Systems (CHES 2010), vol. 6225, S. Mangard and F-X. Standaert, Eds. New York, NY, USA: Springer, 2010, pp. 351-365.

[30] T. Guneysu and C. Paar, "Transforming write collisions in block RAMs into security applications," in Proc. Int. Conf. Field-Programm. Technol. (FPT'09), Dec. 2009, pp. 128-134.

[31] T. Gyorfi O. Cret, and A. Suciu, "High performance true random number generator based on FPGA block RAMs," in Proc. IEEE Int. Symp. Parallel Distrib. Process. (IPDPS'09), May 2009, pp. 1-8.

[32] O. Cret, T. Gyorfi and A. Suciu, "Implementing true random number generators based on high fanout nets," Rom. J. Inf. Sci. Technol., vol. 15, no. 3, pp. 277-298, 2012 [Online]. Available: www.scopus.com

[33] P. Wieczorek, "An FPGA implementation of the resolve time-based true random number generator with quality control," IEEE Trans. Circuits Syst. I: Reg. Papers, vol. 61, no. 12, pp. 3450-3459, Dec. 2014. 
[34] X. Yang and R. C. C. Cheung, "A complementary architecture for high-speed true random number generator," in Proc. Int. Conf. FieldProgramm. Technol. (FPT), Dec. 2014, pp. 248-251.

Honorio Martin received the Ph.D. degree in advanced electronics systems, from Universidad Carlos III de Madrid, Spain, in 2015.

$\mathrm{He}$ is a Postdoctoral Researcher with the Department of Electronics Technology, Universidad Carlos III de Madrid, Madrid, Spain. His research interests include the study of lightweight cryptography hardware implementations, radio-frequency identificatio systems, and lowpower designs.

Pedro Peris-Lopez received the M.Sc. degree in telecommunications engineering and the Ph.D. degree in computer science from Universidad Carlos III de Madrid, Spain, in 2007.

$\mathrm{He}$ is a Visiting Lecturer with the Department of Computer Science, Universidad Carlos III de Madrid, Madrid, Spain. He has authored a great number of papers in specialized journals and conference proceedings on radio-frequency identificatio systems (RFID), and implantable medical devices (IMD). His research interests include protocols design, primitives design, lightweight cryptography, cryptanalysis, RFID, and IMD
Juan E. Tapiador received the M.Sc. and Ph.D. degrees in computer science from the University of Granada, Granada, Spain, in 2000 and 2004, respectively.

$\mathrm{He}$ is an Associate Professor with the Department of Computer Science, Universidad Carlos III de Madrid (UC3M), Madrid, Spain. Between 2009 and 2011, he was Research Associate with the University of York, York, U.K., before joining UC3M. His research interests include applied cryptography, and computer and network security.

Enrique San Millan received the M.Sc. degree in mathematics from La Rioja University, Logroño, Spain, in 1996 and the Ph.D. degree in mathematics engineering from the Universidad Carlos III de Madrid, Madrid, Spain, in 2003.

$\mathrm{He}$ is an Associate Professor with the Department of Electronics Technology, Universidad Carlos III de Madrid. His research interests include hardware design of digital circuits and systems for several field (cryptography, biometry, fault tolerant systems, and communications), and computer assisted design (CAD) tools for design automation and optimization of digital integrated circuits. 